\title{
Interferência dos hábitos nutricionais no perfil metabólico de mulheres com síndrome dos ovários policísticos
}

\author{
Interference from diet on the metabolic profile of women with polycystic ovarian syndrome \\ Milena Martello Gonçalves ${ }^{1}$, Helena Proni Fonseca ${ }^{2}$, Érika Tiemi Fukunaga ${ }^{3}$, José Mendes Aldrighi ${ }^{4}$
}

\section{Resumo}

Objetivo: Avaliar a interferência dos hábitos alimentares no perfil metabólico e antropométrico de mulheres no menacme com Sindrome dos ovários policísticos (SOP). Métodos: Em um estudo transversal, dois grupos de mulheres, um diagnosticado com SOP e o outro não, foram submetidos a testes antropométricos, exames laboratoriais e um questionário validado de frequência de consumo alimentar (QFCA). Foram utilizados os testes estatísticos t-student e Chi quadrado para análise dos resultados. Os grupos "SOP" e "Não SOP" foram comparados em relação aos dados sociodemográficos, antropométricos, metabólicos e dietéticos. Resultados: 62 mulheres completaram as avaliações. Destas, 21 eram mulheres diagnosticadas com SOP e 41 não apresentavam a sindrome. Comparando os parâmetros antropométricos, observamos maiores valores de pressão arterial diastólica $(p=0,005)$, circunferência abdominal $(p=0,027)$ e relação entre circunferência da cintura e do quadril $(p=0,043)$ no grupo com SOP. Com

1. Acadêmica da Faculdade de Ciências Médicas da Santa Casa de São Paulo - $5^{\circ}$ Ano do Curso de Medicina

2. Médica voluntária da Clínica de Ginecologia Endocrinológica, Climatério e Anticoncepção e da Irmandade da Santa Casa de Misericórdia de São Paulo. Mestranda na Faculdade de Saúde Pública da USP

3. Assessora Estatística de Pesquisa Científica na Faculdade de Ciências Médicas da Santa Casa de São Paulo

4. Professor Titular da Faculdade de Ciências Médicas da Santa Casa de São Paulo - Departamento de Obstetrícia e Ginecologia

Trabalho realizado: Irmandade da Santa Casa de Misericórida de São Paulo. Departamento de Ginecologia e Obstetrícia Auxílios, bolsas de estudo: Bolsa de iniciação científica Pibic/CnPQ 2015-2016 para a acadêmica Milena Martello Gonçalves.

Não houve conflito de interesses na realização deste trabalho.

Número de parecer no Comitê de Ética em Pesquisa (CEP) da Santa Casa de São Paulo: 1.051.545. Todas as pacientes consentiram a realização da pesquisa e a divulgação de seus resultados de acordo com a Resolução 196/96 do Conselho Nacional de Saúde.

Endereço para correspondência: Milena Martello Gonçalves. Rua Alcantarilla, 340 - apto 91, Vila Andrade -05717-170 - São Paulo-SP - Brasil.E-mail:milena.martello@gmail.com relação ao perfil metabólico, não houve diferença estatística entre os grupos, considerando glicemia de jejum, TG, LDL e HDL. A avaliação nutricional, por sua vez, revelou que a ingestão de gorduras e fibras foi muito similar em ambos os grupos $(p>0,05)$. Conclusões: O padrão alimentar das voluntárias "SOP" ou "Não SOP" foi semelhante. Assim, nosso estudo não demonstrou influência da dieta no perfil antropométrico e metabólico das mulheres com SOP, sugerindo, portanto, que outros mecanismos, intrínsecos da doença, possam desencadear estas alterações.

Descritores: Síndrome do ovário policístico, Dieta, Metabolismo

\begin{abstract}
Purpose: To evaluate the influence of nutritional habits on metabolic and anthropometric profile of women with Polycystic Ovary Syndrome (PCOS). Methods: This is a cross-sectional study performed on women of childbearing age and diagnosed with PCOS, who were compared to healthy women. Anthropometric and metabolic measurements were made, as well as a validated diet questionnaire was applied. T-student and Chi-square tests were used to compare volunteers with the syndrome to those without PCOS. Results: 62 women completed evaluation; 21 with PCOS and 41 without it. Comparing both groups, "PCOS" women had higher diastolic blood pressure ( $p$ $=0,005)$, waist circumference $(p=0,027)$ and waist/hip ratio $(p=0,043)$. Metabolically, there was no significant difference between them, considering fasting glycemia, triglycerides and cholesterol. The nutritional questionnaire showed that the fiber and lipids consumption were similar $(p>0,05)$ between them. Conclusion: For the "PCOS" and "Non PCOS" groups nutritional analysis, no substantial differences were found. Therefore, our study did not show any influence of diet on metabolic and anthropometric profile of women diagnosed with PCOS. This might be caused by other mechanisms, inherent of the syndrome, than can lead to abnormalities in terms of anthropometry and metabolism.
\end{abstract}

Keywords: Polycystic ovarian syndrome, Diet, Metabolism 


\section{Introdução}

A síndrome dos ovários policísticos (SOP) é o distúrbio endócrino mais prevalente do menacme, acometendo entre 12 e $21 \%$ desta população ${ }^{(1)}$. Metabolicamente, mulheres com SOP manifestam com frequência hiperinsulinismo, resistência insulínica (RI), síndrome metabólica (SM), obesidade, anormalidades no perfil lipídico, predisposição para diabetes tipo 2 (DM II) e doença cardiovascular (DCV) ${ }^{(2)}$. Ademais, são verificados maiores valores séricos de glicemia de jejum, triglicerídeos, LDL, e menores valores de $\mathrm{HDL}^{(2)}$, além de hipersinsulinemia, se comparadas a mulheres não portadoras da síndrome ${ }^{(3)}$.

A fisiopatologia da SOP pode ser entendida através do mecanismo das duas células da esteroidogênese ovariana: mediante o estímulo do $\mathrm{LH}$, as células foliculares da teca convertem colesterol (LDL) em androstenediona e testosterona e, sob ação do FSH, esses androgênios são convertidos a estrona e estradiol pela enzima aromatase, nas células da granulosa ${ }^{(4)}$. Na SOP, os folículos são incapazes de converter o microambiente androgênico para a dominância estrogênica, condição indispensável à ovulação( ${ }^{(4)}$.

Neste aspecto, o hiperandrogenismo é essencial na compreensão da síndrome, porém, além de não estar presente em todas as mulheres, não é o único mecanismo envolvido na fisiopatologia da doença. Também pode ocorrer um defeito na ação da insulina, o que predispõe à hiperinsulinemia e à $\mathrm{RI}^{(5)}$. A RI, por sua vez, pode ocasionar sérias repercussões metabólicas a longo prazo, como DM II, dislipidemia, DCV e o próprio hiperandrogenismo, devido à ação LH-like da insulina nas células da teca ${ }^{(2)}$. Assim como o hiperandrogenismo, a RI não é uma condição obrigatória na $\mathrm{SOP}^{(6)}$. Isto indica a presença de outros mecanismos capazes de gerar o quadro clínico e as complicações metabólicas características nestas pacientes e por isso acredita-se que a SOP seja um conjunto de várias doenças com diferentes fisiopatologias, mas que culminam num quadro clínico semelhante ${ }^{(4)}$.

Não obstante, a obesidade, atualmente encontrada em proporções epidêmicas na população, atua como fator predisponente ao desenvolvimento da $\mathrm{SOP}^{(7)}$. Dessa forma, é esperado que conforme a população torna-se mais obesa, a SOP também seja mais comum $^{(7)}$. O percentual de mulheres com a síndrome que apresenta obesidade é próximo de $50 \%{ }^{(5)}$, sendo que alguns estudos já avaliaram a frequência de irregularidades no metabolismo em mulheres acima do peso com e sem SOP, sendo observada maior porcentagem de gordura total, hemoglobina glicada, glicemia em jejum e triglicérides naquelas com a síndrome ${ }^{(8)}$. Além disso, a relação insulina/hiperinsulinemia é maior em mulheres com a doença ${ }^{(7)}$. A resistência à insulina é intrínseca à síndrome, sendo que a obesidade, principalmente obesidade central, pode exacerbá-la(9).

O manejo da SOP está intimamente relacionado a uma mudança de estilo de vida ${ }^{(10)}$, com perda ponderal, para melhora de sintomas clínicos da SOP, sendo que uma perda de $5 \%$ do peso corporal pode ser capaz de reduzir a resistência insulínica, o hiperandrogenismo, a irregularidade menstrual e a infertilidade ${ }^{(11)}$ . Todavia, um número escasso de estudos avaliou a influência dos hábitos nutricionais no perfil metabólico das pacientes identificadas com SOP. A comparação entre mulheres com e sem a síndrome, de IMC normal (IMC $\left.<25 \mathrm{~kg} / \mathrm{m}^{2}\right)$, revela que as portadoras da SOP apresentam dieta com menor ingestão de carboidratos, proteínas, colesterol, caloria e gordura total. Se a mesma análise for feita com um grupo de IMC mais elevado (IMC $>25 \mathrm{~kg} / \mathrm{m}^{2}$ ), não há diferenças significativas entre o consumo energético ou de macronutrientes ${ }^{(12)}$. Entretanto, não há publicações a respeito desses diferentes padrões dietéticos e sua relação com o perfil metabólico. Assim, poderíamos determinar a ação, não apenas do excesso de gordura corpórea, mas, ainda, do padrão alimentar na alteração dos padrões das variáveis metabólicas.

Portanto, a suprir a escassez de estudos nesse tema, o presente projeto avaliou a influência dos hábitos nutricionais no perfil metabólico em pacientes com SOP, independetemente do IMC.

\section{Material e Métodos}

Foi realizado um estudo transversal no Departamento de Ginecologia e Obstetrícia da Santa Casa de Misericórdia de São Paulo (DOGI), em mulheres entre 15 a 45 anos, diagnosticadas com SOP e não diagnosticadas com a doença.

A SOP foi definida pelos critérios de Rotterdam de 2003, que devem incluir duas ou três características citadas a seguir: 1 . Irregularidade menstrual ou amenorréia; 2. Hiperandrogenismo clínico e/ou laboratorial; 3. Morfologia ovariana policística ao ultrassom realizado entre o terceiro e o quinto dia do ciclo menstrual, definida pela presença de 12 ou mais folículos de 2-9 $\mathrm{mm}$ e/ou volume ovariano superior a $10 \mathrm{ml}$ em pelo menos um dos ovários. Além disso, foram excluídos diagnósticos diferenciais como Hiperprolactinemia, Hiperplasia Adrenal Congênita, Síndrome de Cushing ou gestação.

Foram comparados dados antropométricos, incluindo pressão arterial (PA), circunferência abdominal (CA), circunferência de quadril (CQ), peso, altura e IMC. A pressão arterial (PA) foi aferida, após 15 minutos de repouso, com a voluntária sentada e seu braço apoiado ao nível do coração. A CA foi medida, por uma fita métrica, na porção média da região com- 
preendida pela borda inferior da costela e pela crista ilíaca. ACQ foi verificada, também por fita métrica, na altura de maior circunferência das nádegas. O peso e a altura foram obtidos em balança marca Toledo, modelo digital 2096PP/2, série 04068013799-kf, apoiada sobre uma superfície plana, lisa e firme, com precisão de $50 \mathrm{~g}$, medindo um peso máximo de $200 \mathrm{~kg}$ e um mínimo de $1 \mathrm{~kg}$. A mulher era posicionada no centro do equipamento, com o mínimo de roupa possível, descalça, ereta, com os pés juntos e os braços estendidos ao longo do corpo, sendo mantida nessa posição até que as leituras sejam realizadas. O IMC foi calculado pela razão do peso $(\mathrm{kg})$ pela altura $(\mathrm{m})$ ao quadrado. De acordo com o Ministério da Saúde, IMC entre 18,5 e $24,9 \mathrm{~kg} / \mathrm{m}^{2}$ indica peso adequado, entre 25 e 29,9 , sobrepeso e igual ou maior que $30 \mathrm{mg} / \mathrm{m}^{2}$, obesidade.

Exames laboratoriais, glicemia de jejum, triglicerídeos, HDL colesterol, LDL colesterol foram coletados no Laboratório Central do complexo hospitalar da Santa Casa de Misericórdia de São Paulo. A glicose, triglicerídeos e HDL colesterol foram determinados por métodos colorimétrico-enzimáticos utilizando o COBAS INTEGRA 400 PLUS da Roche Diagnostics (Mannheim, Alemanha). O LDL colesterol é estimado indiretamente utilizando: $\mathrm{LDL}=$ colesterol total $-\mathrm{HDL}$ - triglicerídios/5.

Ademais, todas as mulheres responderam a um questionário de frequência de Consumo alimentar (QFCA), validado em português ${ }^{(13)}$, e aplicados por um examinador. (Figura 1)

Todas mulheres participaram voluntariamente do estudo, conduzido de acordo com a Declaração de Helsinque ${ }^{(14)}$ e aprovado pelo Comitê de Ética em
Pesquisa (CEP) da Irmandade da Santa Casa de São Paulo (parecer número 1.051.545), após assinar termo de consentimento livre e esclarecido, podendo retirar-se do estudo a qualquer momento, sem prejuízo ao seu tratamento. Apenas foram excluídas voluntárias usuárias de anticoncepcionais hormonais ou outros medicamentos; diagnosticadas com diabetes tipo I ou outros distúrbios metabólicos.

Os dados foram tabulados no Microsoft Excel como média, mais ou menos desvio padrão e analisados por meio de testes $t$-student e Chi quadrado ou Mann-whitney, de acordo com o número amostral e a disparidade de dados na amostra. Foram considerados significantes quando menor que 0,05 , ou seja, com intervalo de confiança de $95 \%$. Os grupos "SOP" e "Não SOP" foram comparados em relação aos dados sociodemográficos, antropométricos, metabólicos e dietéticos. Posteriormente, repetimos a análise dos hábitos alimentares categorizando o consumo de gorduras e fibras como adequado e inadequado para todas as pacientes do estudo, e depois apenas para o grupo SOP e apenas para o controle.

\section{Resultados}

Foram recrutadas 138 voluntárias, dos Ambulatórios de Ginecologia e Obstetrícia da Santa Casa de São Paulo, após assinatura do Termo de Consentimento Livre e Esclarecido. Destas, três foram excluídas por Diabetes mellitus tipo I, Síndrome de Swyer e insuficiência renal crônica dialítica. Das 135 mulheres restantes, 41 faziam parte do grupo com SOP e 87 do grupo controle. Foram coletados dados antropométricos, nutricionais,

\begin{tabular}{|c|c|c|c|c|c|}
\hline Alimento & Não Come (0) & menos de $1 \mathrm{x} /$ mês $(0)$ & - 2 a 3x/mês (1) & 1 a 2 x/semana (2) 3 a $4 x$ na semana (3) & 5 ou mais $x /$ semana $(4)$ \\
\hline \multicolumn{6}{|c|}{ ( } \\
\hline \multicolumn{6}{|l|}{ Frngo Frito } \\
\hline \multicolumn{6}{|l|}{ Salsicha e Linguiça } \\
\hline \multicolumn{6}{|l|}{ Frios } \\
\hline \multicolumn{6}{|l|}{ Maionese } \\
\hline \multicolumn{6}{|l|}{ Manteiga ou Margarina } \\
\hline \multicolumn{6}{|l|}{ Ovos } \\
\hline \multicolumn{6}{|l|}{ Bacon } \\
\hline \multicolumn{6}{|l|}{ Queijos e requeijão } \\
\hline \multicolumn{6}{|l|}{ Leite Integral } \\
\hline \multicolumn{6}{|l|}{ Batata Frita } \\
\hline \multicolumn{6}{|l|}{ Salgadinhos de pacote } \\
\hline \multicolumn{6}{|l|}{ Produtos de pastelaria } \\
\hline \multicolumn{6}{|l|}{ Sucos naturais de frutas } \\
\hline \multicolumn{6}{|l|}{ Frutas } \\
\hline \multicolumn{6}{|l|}{ Verduras } \\
\hline \multicolumn{6}{|l|}{ Batata Frita } \\
\hline \multicolumn{6}{|l|}{ Feijão, lentilha, ervilha } \\
\hline \multicolumn{6}{|l|}{ Legumes } \\
\hline \multicolumn{6}{|l|}{ Careais integrais } \\
\hline \multicolumn{6}{|l|}{ Pão integral } \\
\hline \multicolumn{6}{|l|}{ Pães convencionais } \\
\hline Café & & & & & \\
\hline
\end{tabular}

Figura 1 - QFCA validado em português, utilizado no estudo 
sociodemográficos, além de exames laboratoriais, que deveriam ser agendados no Laboratório Central. Desse grupo, apenas 62 mulheres compareceram para coleta de exames, totalizando 21 do grupo SOP e 41 do grupo controle, sendo as demais excluídas.

As participantes do grupo não-SOP, foram em média mais velhas e apresentavam renda familiar mais elevada que o grupo SOP. Comparando os parâmetros antropométricos entre os dois grupos, observamos maiores valores de PAD, CA e C/Q nas mulheres com SOP. Quanto ao IMC e à PAS não houve diferença significante entre os grupos (Tabela 1). Com relação ao perfil metabólico, também não houve diferença estatística entre os grupos, considerando glicemia de jejum, TG, LDL e HDL.

A avaliação nutricional das mulheres "SOP" vs "Não SOP", principal objetivo do estudo, demostrou ingestão muito semelhante de gorduras e fibras (Tabela 2) nos dois grupos, independentemente do diagnóstico de SOP.

Posteriormente, categorizamos a ingestão de gorduras e fibras, das 62 mulheres, como adequado ou inadequado, de acordo com a validação do questionário aplicado neste estudo ${ }^{(13)}$, e repetimos a análise estatística para as variáveis antropométricas e metabólicas. Não houve diferença relevante. A dieta dos grupos de mulheres no estudo é semelhante na análise da adequação do consumo de gorduras e fibras. (Tabelas 3 e 4)
Por fim, a ingestão de gorduras foi avaliada especificamente no grupo SOP e no grupo controle. Mais uma vez, não houve diferença estatística relevante. Ou seja, a dieta das voluntárias do estudo, no que diz respeito a ingesta adequada/inadequada de gordura, não varia de forma significante e não esteve associada aos parâmetros antropométricos. $\mathrm{O}$ mesmo não foi repetido também para o consumo de fibras, visto que o número de pacientes com ingesta inadequada desse nutriente foi insuficiente.

\section{Discussão}

É consenso na literatura que mulheres com SOP apresentam pior perfil antropométrico ${ }^{(8)}$,em relação às não portadoras, o que também foi encontrado no presente estudo, onde as pacientes com a síndrome apresentaram maiores valores de $\mathrm{PAD}, \mathrm{CA}$ e relação $\mathrm{C} / \mathrm{Q}$. Os demais parâmetros antropométricos e metabólicos foram estatisticamente semelhantes, provavelmente devido à amostra reduzida, consequente à exclusão de pacientes que não compareceram à coleta laboratorial.

De acordo com a Internacional Diabetes Federation, uma circunferência abdominal maior que $80 \mathrm{~cm}$, em mulheres, indica obesidade central ${ }^{(15)}$ e está associada à $\mathrm{RI}^{(16)}$. De fato, a RI, com consequente hiperinsulinemia, é verificada com frequência em pacientes com SOP (2), podendo explicar parte da fisiopatologia da doença ${ }^{(4)}$.

De forma semelhante, o aumento da relação C/Q Tabela 1

\begin{tabular}{|c|c|c|c|c|c|}
\hline & \multicolumn{2}{|c|}{$\operatorname{SOP}(N=21)$} & \multicolumn{2}{|c|}{ Não $\operatorname{SOP}(N=41)$} & \multirow[t]{2}{*}{$p$} \\
\hline & Média $(D P)$ & IC $(95 \%)$ & Média $(D P)$ & IC $(95 \%)$ & \\
\hline Idade & $28,5(1,3)$ & $25,8-31,2$ & $33,9(1,3)$ & $31,6-36,6$ & $0,013^{*}$ \\
\hline Renda (R\$) & $1980(242)$ & $1473,3-2486,6$ & $4257(456,7)$ & $2726,3-5787,7$ & $0,040^{*}$ \\
\hline IMC & $29,6(7,9)$ & $26,1-33,2$ & $26,8(5,0)$ & $25,2-28,4$ & 0,088 \\
\hline PAS (mmHg) & $116,7(20,3)$ & $107,4-125,9$ & $110,3(13,1)$ & $106,1-114,4$ & 0,140 \\
\hline PAD (mmHg) & $78,9(12,1)$ & $73,4-84,5$ & $70,7(9,8)$ & $67,6-73,8$ & $0,005^{*}$ \\
\hline $\mathrm{CA}(\mathrm{cm})$ & $96,8(15,7)$ & $89,6-103,9$ & $88,8(11,7)$ & $85,1-92,5$ & $0,027^{*}$ \\
\hline $\mathrm{CQ}(\mathrm{cm})$ & $107,9(12,8)$ & $102,1-113,7$ & $104,4(9,9)$ & $101,3-107,5$ & 0,232 \\
\hline $\mathrm{C} / \mathrm{Q}$ & $89,8(9,0)$ & $85,7-93,9$ & $85,0(8,3)$ & $82,4-87,7$ & $0,043^{*}$ \\
\hline
\end{tabular}

IMC: índice de massa corpórea / PAS: pressão arterial sistólica / PAD: pressão arterial diastólica / CA: circunferência abdominal / CQ: circunferência de quadril / C/Q: circunferência abdominal / circunferência de quadril

Tabela 2

\begin{tabular}{|c|c|c|c|c|c|}
\hline \multicolumn{6}{|c|}{ Tabela 2} \\
\hline & \multicolumn{2}{|c|}{$\operatorname{SOP}(N=21)$} & \multicolumn{2}{|c|}{ Não SOP $(N=41)$} & \multirow[b]{2}{*}{$p$} \\
\hline & Média (DP) & IC $(95 \%)$ & Média (DP) & IC $(95 \%)$ & \\
\hline Gorduras (g) & $75,3(18,1)$ & $66,8-83,7$ & $73,0(15,9)$ & $67,9-78,0$ & 0.616 \\
\hline Fibras (g) & $15,6(4,0)$ & $13,9-17,4$ & $15,8(5,7)$ & $14,0-17,6$ & 0.862 \\
\hline
\end{tabular}


Dados antropométricos de acordo com o consumo de gorduras em "Adequado" vs "Inadequado"

\begin{tabular}{|c|c|c|c|c|c|}
\hline & \multicolumn{2}{|c|}{ Adequado $(N=36)$} & \multicolumn{2}{|c|}{ Inadequado $(N=25)$} & \multirow{2}{*}{$p$} \\
\hline & Média $(D P)$ & IC $(95 \%)$ & Média (DP) & IC $(95 \%)$ & \\
\hline IMC & $26,2(1,2)$ & $23,5-28,9$ & $28,54(0,9)$ & $26,5-30,53$ & 0,15 \\
\hline PAS (mmHg) & $112,3(4,2)$ & $103,3-121,5$ & $111,9(2,2)$ & $107,4-116,5$ & 0,853 \\
\hline PAD (mmHg) & $68,4(2,4)$ & $63-73,9$ & $74,1(1,6)$ & $70,9-77,7$ & 0,112 \\
\hline $\mathrm{CA}(\mathrm{cm})$ & $87,8(2,5)$ & $82,5-93,2$ & $93,5(2,3)$ & $88,8-98,1$ & 0,110 \\
\hline $\mathrm{CQ}(\mathrm{cm})$ & $103,6(2,2)$ & $98,8-108,4$ & $106,2(1,5)$ & $103,0-109,4$ & 0,440 \\
\hline $\mathrm{C} / \mathrm{Q}$ & $0,84(0,2)$ & $0,79-0,89$ & $0,86(0,1)$ & $0,84-0,89$ & 0,392 \\
\hline
\end{tabular}

IMC: índice de massa corpórea / PAS: pressão arterial sistólica / PAD: pressão arterial diastólica / CA: circunferência abdominal / CQ: circunferência de quadril / C/Q: circunferência abdominal / circunferência de quadril

Tabela 4

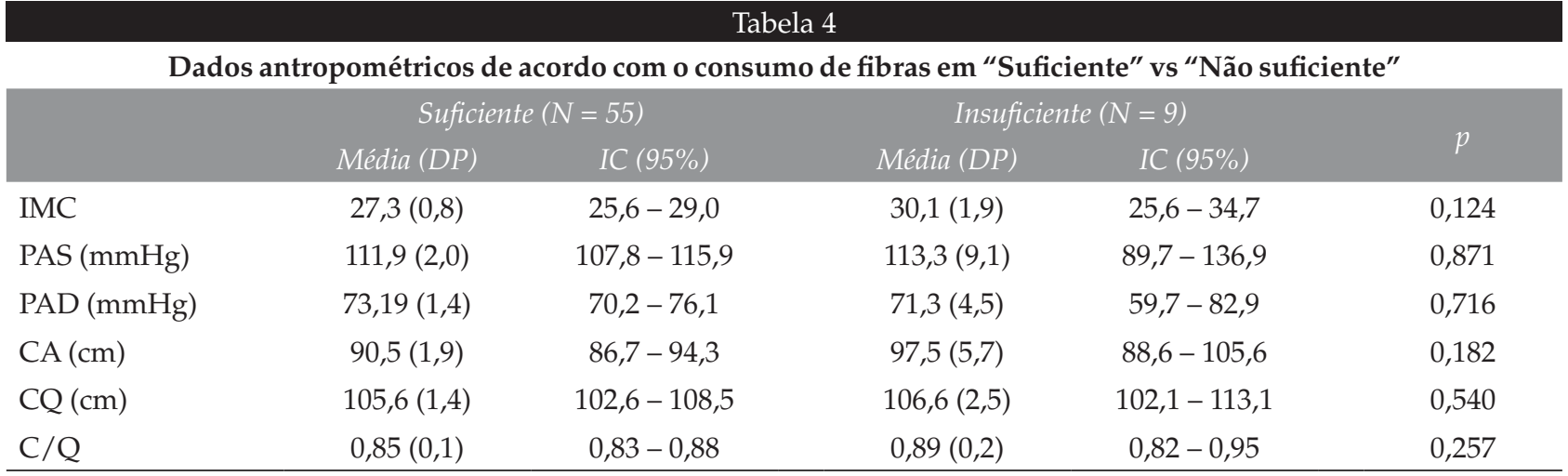

IMC: índice de massa corpórea / PAS: pressão arterial sistólica / PAD: pressão arterial diastólica / CA: circunferência abdominal / CQ: circunferência de quadril / C/Q: circunferência abdominal / circunferência de quadril

( $C / Q>0,8$ em mulheres) é indicativo de $R C V$, sendo esse o índice de distribuição de gordura corporal mais utilizado em pesquisa epidemiológica. Cinturas estreitas e quadris largos estão associados à proteção contra doenças cardiovasculares. Já quadris estreitos podem representar quantidade reduzida de massa muscular, desencadeando menor atividade da insulina e da lipase lipoprotéica, com concomitante redução na utilização dos ácidos graxos pelas células musculares. Em contrapartida, quadris largos apresentam maior concentração da lipase lipoprotéica devido à maior quantidade de tecido muscular. Além disso, há menor turnover de ácidos graxos no tecido adiposo gluteofemoral em relação ao tecido adiposo visceral, o que favorece a sensibilidade insulínica ${ }^{(16)}$.

O manejo da SOP, com suas repercussões antropométricas e metabólicas, deve incluir perda ponderal e atividade física ${ }^{(10)}$, porém há pouca investigação sobre a importância isolada da dieta. Trabalhos publicados que avaliam pacientes com SOP do ponto de vista nutricional são, em sua maioria, amostras pequenas (17), com atividade física concomitante ${ }^{(18)}$ ou estudos em animais ${ }^{(19)}$, sem associação com dados metabólicos. Esta pesquisa, por sua vez, teve o objetivo de correla- cionar variáveis antropométricas e metabólicas à dieta das pacientes "SOP" e "Não SOP". No nosso caso, a ingestão de gorduras e fibras foi muito semelhante em ambos os grupos. Ou seja, independentemente da doença, o consumo desses macronutrientes é similar e sem associação direta com padrões antropométricos ou metabólicos. Um dos únicos resultados que pode ser equiparado a esse é do estudo caso-controle publicado no Global Journal of Health Sciences em 2016, com 142 mulheres com SOP e 140 sem a síndrome, no qual foi observado que o consumo de macronutrientes é, de fato, muito semelhante entre um grupo de pacientes "SOP" e outro "sem SOP" (10,12).

No presente estudo categorizamos a ingestão de fibras e gorduras de todas as 62 mulheres em "Adequado" ou "Inadequado", de acordo com o próprio artigo de validação do questionário utilizado ${ }^{(13)}$, e repetimos os cálculos para as variáveis antropométricas e metabólicas. No caso do consumo de fibras, apenas nove voluntárias obtiveram ingesta classificada como inadequada, um fator limitante para a análise estatística, devido à amostra reduzida. Não houve diferença estatística para qualquer uma das variáveis antropométricas ou metabólicas, se compararmos 
aquelas que consomem fibras adequadamente com aquelas com ingesta inadequada. Quanto à ingestão de gordura, 25 mulheres tiveram consumo inadequado e 36 adequado, também não havendo diferenças entre os grupos nos parâmetros supracitados. Portanto, a classificação do consumo de fibras, e também de gorduras, como adequado/inadequado não esteve associada às variáveis antropométricas e metabólicas.

Essa investigação, com base na literatura disponível, verificou portanto, que independentemente da ingestão adequada/inadequada de fibras e gorduras, a paciente com SOP tende a apresentar pior perfil antropométrico. Ou seja, o padrão alimentar é insuficiente para explicar a anormalidade antropométrica das mulheres com a síndrome, visto que ambos os grupos, "SOP" e "Não SOP", consomem fibras e gorduras de forma semelhante. Assim, nosso estudo não demonstrou influência da dieta no perfil antropométrico das pacientes com SOP, sugerindo, portanto, que outros mecanismos, intrínsecos da doença, possam desencadear estas alterações.

Análises de padrões metabólicos e nutricionais devem considerar a idade e a renda familiar dos participantes, uma vez que a idade está associada a modificações metabólicas e a renda à capacidade de compra dos alimentos ${ }^{(13)}$. Entretanto, no presente trabalho, ambas as variáveis idade e renda foram estatisticamente diferentes entre os grupos "SOP" e "Não SOP", sendo as mulheres com SOP em média mais novas e com menor renda. Porém, com o avançar da idade, é esperado uma piora de padão antropométrico e metabólico, o que se constituiu num viés. Portanto, novos estudos que pareiem voluntárias por idade, renda familiar e incluam a avaliação de outros macronutrientes, como carboidratos, trariam informações mais consistentes. Em uma amostra de mulheres com idade semelhantes, aquelas com SOP provavelmente apresentariam piores índices antropométricos, como já tem indicado a literatura disponível ${ }^{(8)}$. Além disso, ampliar o número total de voluntárias, bem como o número de mulheres com dieta inadequada, seria extremamente válido para a melhor compreensão das irregularidades metabólicas da SOP.

Como conclusão, as mulheres com SOP possuem pior perfil antropométrico e metabólico do que mulheres saudáveis, porém, não há relação destes parâmetros com a alimentação, sugerindo que mecanismos intrínsecos à doença possam justificar essas alterações. Há necessidade de novos estudos de melhor poder estatístico para confirmar estas informações.

\section{Referências}

1. March WA, Moore VM, Willson KJ, Phillips DI, Norman RJ, Davies MJ. The prevalence of polycystic ovary syndrome in a community sample assessed under contrasting diagnostic criteria. Hum Reprod. 2010; 25(2):544-51.

2. Shorakae S, Boyle J, Teede H. Polycystic ovary syndrome: a common hormonal condition with major metabolic sequelae that physicians should know about. Intern Med J. 2014; 44(8):720-6.

3. Wild RA. Dyslipidemia in PCOS. Steroids. 2012;77(4):295-9.

4. Fritz MA, Speroff L. Clinical gynecologic endocrinology and infertility. Baltimore: William \& Wilkins; 1995. 1488p.

5. Messinis IE, Messini CI, Anifandis G, Dafopoulos K. Polycystic ovaries and obesity. Best Pract Res Clin Obstet Gynaecol. 2015; 29(4):479-88.

6. Rodrigues de Oliveira Penaforte F, Cremonezi Japur C, DíezGarcía RW, Salles Macedo C, García Chiarello P. The use of body circumferences for the prediction of intra-abdominal fat in obese women with polycystic ovary syndrome. Nutr Hosp. 2012; 27(5):1662-6.

7. Motta AB. The role of obesity in the development of polycystic ovary syndrome. Curr Pharm Des. 2012; 18(17):2482-91.

8. Kandaraki E, Christakou C, Diamanti-Kandarakis E. Metabolic syndrome and polycystic ovary syndrome... and vice versa. Arq Bras Endocrinol Metabol. 2009; 53(2):227-37.

9. Leão LMCSM. Obesidade e síndrome dos ovários policísticos: vínculo fisiopatológico e impacto no fenótipo das pacientes. Rev HUPE.2014; 13(1):33-7.

10. Sedighi S, Amir Ali Akbari S, Afrakhteh M, Esteki T, Alavi Majd H, Mahmoodi Z. Comparison of lifestyle in women with polycystic ovary syndrome and healthy women. Glob J Health Sci. 2014; 7(1):228-34.

11. Rondanelli M, Perna S, Faliva M, Monteferrario F, Repaci E, Allieri F. Focus on metabolic and nutritional correlates of polycystic ovary syndrome and update on nutritional management of these critical phenomena. Arch Gynecol Obstet. 2014; 290(6):1079-92.

12. Shishehgar F, Ramezani Tehrani F, Mirmiran P, Hajian S, Baghestani AR, Moslehi N. Comparison of dietary intake between polycystic ovary syndrome women and controls. Glob J Health Sci. 2016; 8(9):54801.

13. Ribeiro AC, Sávio KEO, Rodrigues MLCF, Costa THM, Schmitz BAS. Validação de um questionário de frequência de consumo alimentar para população adulta. Rev Nutr. 2006; 19(5):553-62.

14. Associação Médica Mundial. Declaração de Helsinki. [online]. Disponível em: http:/ /www.fcm.unicamp.br/fcm/sites / default/files/declaracao_de_helsinque.pdf (21 mai 2017)

15. International Diabetes Federation. The IDF consensus worldwide definition of the diabetes. [online]. Available from: https: / /www.idf.org/e-library/consensus-statements / 60idfconsensus-worldwide-definitionof-the-metabolic-syndrome (2017 May 2)

16. Vasques AC, Rosado L, Rosado G, Ribeiro RC, Franceschini $\mathrm{S}$, Geloneze $\mathrm{B}$. Indicadores antropométricos de resistência à insulina. Arq Bras Cardiol. 2010; 95(1): e14-e23.

17. Goss AM, Chandler-Laney PC, Ovalle F, Goree LL, Azziz R, Desmond RA, et al. Effects of a eucaloric reduced-carbohydrate diet on body composition and fat distribution in women with PCOS. Metabolism. 2014; 63(10):1257-64.

18. Love JG, McKenzie JS, Nikokavoura EA, Broom J, Rolland C, Johnston KL. The experiences of women with polycystic ovary syndrome on a very low-calorie diet. Int J Womens Health. 2016; 8:299-310.

19. Ressler IB, Grayson BE, Ulrich-Lai YM, Seeley RJ. Diet-induced obesity exacerbates metabolic and behavioral effects of polycystic ovary syndrome in a rodent model. Am J Physiol Endocrinol Metab. 2015; 308(12):E1076-84.

Trabalho recebido:20/05/2017

Trabalho aprovado: 21/03/2018 